\title{
Queilite actínica associada a anemia ferropriva em idoso: relato de caso
}

\author{
Acetinique cheilitis associated with anemia iron-deficiency elderly: case report
}

Queilite actinica asociada la anemia ferropriva en idoso: relato de caso

Helissa Mayane Nunes da Silva Oliveira ${ }^{1 *}$, Pedro Henrique Barros Alécio ${ }^{1}$, lago Fernando Rodrigues Gomes ${ }^{1}$, Catarina Rodrigues Rosa de Oliveira ${ }^{1}$, Sonia Maria Soares Ferreira ${ }^{1}$

\section{RESUMO}

Objetivo: O objetivo desse relato é demostrar a importância da estomatologia, onde a busca dos fatores etiológicos seja local ou sistêmico são necessários para o correto diagnóstico e tratamento dos pacientes. Métodos: Os dados foram obtidos por meio de revisão do prontuário, entrevista com o paciente, registro fotográfico dos métodos diagnósticos aos quais o paciente foi submetido e revisão da literatura. Resultados: A queilite actínica é uma lesão cutânea pré-maligna comum, causada pela radiação ultravioleta cumulativa na pele exposta ao sol, especialmente nas pessoas de pele clara. Relata-se o caso de um paciente do sexo masculino, 60 anos de idade, leucoderma, que procurou o serviço de Estomatologia, queixando-se de lesão dolorosa em região de lábio inferior há aproximadamente dois meses e foi diagnosticado com Queilite Actínica associada a anemia ferropriva. Foi realizado tratamento para a anemia e para queilite e ocorreram cicratização das lesões. Conclusão: Por fim, ressalta-se a importância da busca dos fatores etiológicos seja local ou sistêmico para o completo tratamento de lesões bucais.

Palavras-chave: Queilite, Lesões Pré-Cancerosas, Anemia Ferropriva.

\begin{abstract}
Objective: The objective of this report is to demonstrate the importance of stomatology, where the search for etiological factors is local or systemic are necessary for the correct diagnosis and treatment of patients. Methods: The information was obtained by reviewing the medical record, interviewing the patient, photographic record of the diagnostic methods to which the patient was submitted and review of the literature. Results: Actinic cheilitis is a common premalignant cutaneous lesion caused by cumulative ultraviolet radiation on the skin exposed to the sun, especially in light-skinned people. We report the case of a 60-year-old male patient, leucoderma, who sought out the stomatology service complaining of a painful injury in the lower lip region for approximately two months and was diagnosed with actinic cheilitis associated with iron deficiency anemia. Treatment was performed for anemia and cheilitis and cicatrization of the lesions occurred. Conclusion: Finally, the importance of the search for etiological factors, whether local or systemic, for the complete treatment of oral lesions is emphasized.
\end{abstract}

Keywords: Cheilits, Precancerous Conditions, Anemia.

${ }^{1}$ Centro Universitário Cesmac. *E-mail: helissanunes@gmail.com

SUBMETIDO EM: 6/2018 | ACEITO EM: 7/2018 | PUBLICADO EM: 12/2018 


\section{RESUMEN}

Objetivo: El objetivo de este relato es demostrar la importancia de la estomatología, donde la búsqueda de los factores etiológicos sea local o sistémico son necesarios para el correcto diagnóstico y tratamiento de los pacientes. Métodos: Los dados fueron obtenidos por medio de revisión del prontuario, entrevista con el paciente, registro fotográfico de los métodos diagnósticos a los cuales el paciente fue sometido y revisión de la literatura. Resultados: La queilitis actínica es una lesión cutánea premaligna común, causada por la radiación ultravioleta acumulativa en la piel expuesta al sol, especialmente en las personas de piel clara. Se relata el caso de un paciente del sexo masculino, 60 años de edad, leucoderma, que buscó el servicio de estomatología, quejándose de lesión dolorosa en región de labio inferior hace aproximadamente dos meses y fue diagnosticado con Queilitis Actínica asociada la anemia ferropénica. Se realizó tratamiento para la anemia y para la queilitis y ocurrió cicatrización de las lesiones. Conclusión: Por último, se resalta la importancia de la búsqueda de los factores etiológicos sea local o sistémico para el completo tratamiento de lesiones bucales.

Descriptores: Queilitis, Lesiones Precancerosas, Anemia Ferropénica.

\section{INTRODUÇÃO}

A queilite actínica é uma lesão cutânea pré-maligna comum, causada pela radiação ultravioleta cumulativa na pele exposta ao sol, especialmente nas pessoas de pele clara e acima de 40 anos (NEVILLE et al., 2009; REGEZI e SCIUBBA, 2000). Essa lesão acontece principalmente no sexo masculino devendo-se ao fato de que muitos homens trabalham, por períodos longos, sob exposição solar, sendo o lábio inferior o mais afetado em razão de sua anatomia (MARTINS et al., 2007).

Os lábios ficam rugosos, ressecados e ásperos, com o passar do tempo pode surgir algumas estrias ou fissuras próximo ao limite da pele e vermelhão. Alguns sintomas são frequentes como a sensação de secura e de descamação, também pode está presente a perda da linha que delimita a parte avermelhada do lábio e a pele (PIÑERA-MARQUES et al., 2010; BERTINI, 2004; ROSSOE et al., 2011).

Neste relato apresenta-se como fatores etiológicos da lesão, a associação da exposição solar com a anemia ferropriva. A anemia, por sua vez, tem como seu principal sinal bucal a palidez da mucosa, esta foi encontrada tanto na histórica clínica do paciente como por seus sinais bucais (POFFO et al, 2008).

A anemia tem sido definida como a redução patológica da concentração de hemoglobina $(\mathrm{Hb})$ circulante, desencadeada por mecanismos fisiopatológicos diversos. A anemia é o problema hematológico mais comumente encontrado nos indivíduos idosos (BARBOSA et al., 2006).

O diagnóstico diferencial da anemia, com o objetivo de caracterizar o tipo de anemia na população idosa, pode ser realizado com exames clínicos e laboratoriais que incluam os indicadores hematológicos: hemoglobina $(\mathrm{Hb})$, hematócrito $(\mathrm{Hct})$ e hemácias $(\mathrm{He})$ e os indicadores hematimétricos: volume corpuscular médio (VCM), hemoglobina corpuscular média (HCM), concentração de hemoglobina corpuscular média $(\mathrm{CHCM})$ e amplitude de distribuição dos eritrócitos (RDW) (BARBOSA et al., 2006).

Os exames para o correto diagnostico deve-se tomar como base os achados clínicos e histopatológicos. Mas pode ocorrer, divergências para à necessidade de biópsia (MIRANDA, FERRARI, CALANDRO, 2011; TOMMASI,2002).

O objetivo desse relato é demostrar a importância da estomatologia, onde a busca dos fatores etiológicos seja local ou sistêmico são necessários para o correto diagnóstico e tratamento dos pacientes. 


\section{RELATO DE CASO}

Paciente do gênero masculino, 60 anos de idade, leucoderma (Figura 1a), foi ao Posto de Atendimento Médico Salgadinho, em Maceió, Alagoas, no bloco de Estomatologia, queixando-se de lesão dolorosa em região de lábio inferior há aproximadamente dois meses.

Em sua história médica, havia relato do aparecimento de lesões semelhantes em 2006, cujo diagnóstico havia sido de queilite actínica associada a anemia ferropriva. O paciente foi acompanhado até 2011 . Em março de 2015, o mesmo retorna apresentando lesões ulceradas em lábio inferior (Figura 1a).

Ao exame extra-oral, observou-se a presença de lesão ulcerada, além de regiões ásperas, placas leucoplásicas e perda da linha que separa o vermelhão do lábio da pele (Figura 1b). Na comissura, bilateral, foram observadas fissuras irradiadas, com sintomatologia dolorosa (Figura 1a).

Os diagnósticos clínicos das lesões foram, queilite actínica e angular, respectivamente. Diante da história prévia de anemia ferropriva foi solicitado hemograma completo com contagem de plaquetas. Foi prescrito Bepantol Derma e protetor labial. O resultado do hemograma confirmou anemia e foi prescrito suplementação de ferro e vitaminas. O paciente retornou um mês após a suplementação com novo hemograma ainda sem correção do quadro anêmico. O paciente foi encaminhado para hematologia para investigação e correção do quadro anêmico. As lesões só cicatrizaram por completo após tratamento da anemia (Figura $\underline{2 a}$ e $\underline{2 b}$ ) e 0 paciente continua sendo acompanhado clinicamente até hoje, onde não ocorreram recorrências das lesões.

Figura 1 - Lesões ulceradas em lábio inferior

Fig. 1a: Aspecto extra oral evidenciando alteração no lábio inferior. Comissura labial com fissuras irradiadas.
Fig. 1b: Paciente apresentando lesões ulceradas, associada à placas leucoplásicas e apagamento da linha que separa o vermelhão do lábio da pele do mento.
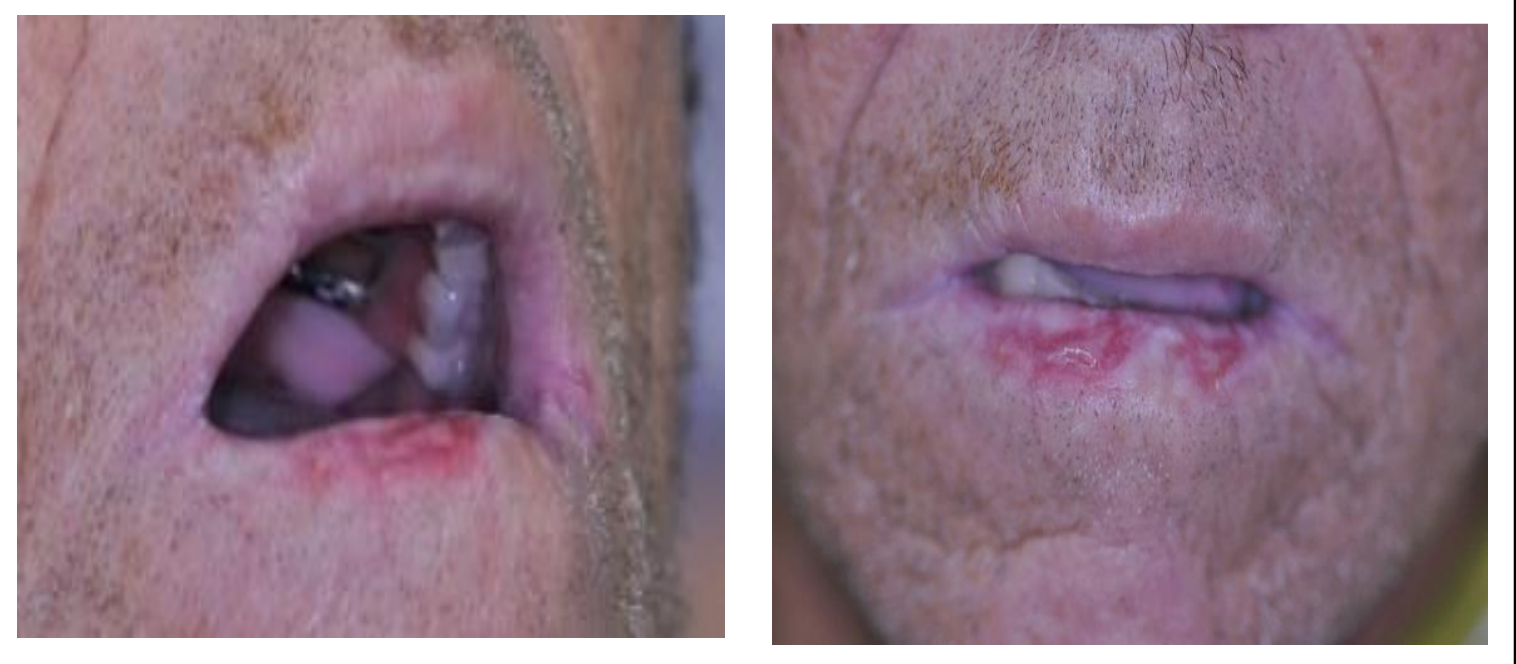
Figura 2 - Processo de cicatrizaração no lábio inferior

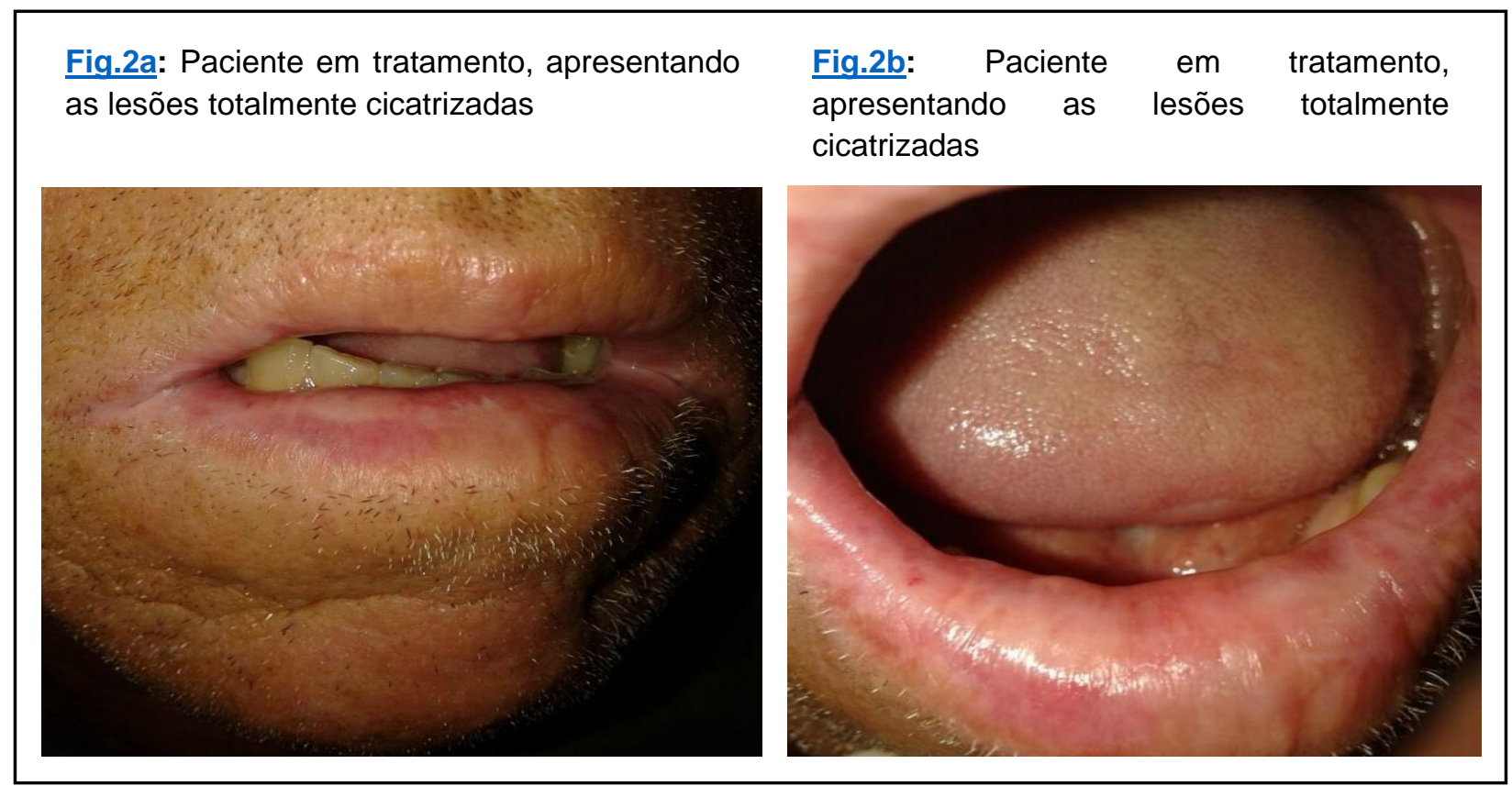

\section{DISCUSSÃO}

A literatura descreve que a queilite actínica (QA) tem predileção pelo gênero masculino, principalmente entre os 40 e 50 anos de idade, assim como, associação com a exposição solar, tabagismo e etilismo (CINTRA et al., 2013).

Alguns estudos apontaram que o gênero feminino é o menos acometido, uma vez que, o uso de batom pode fornecer um determinado grau de proteção labial (CAVALCANTE et al., 2008) .

Segundo Nico, et al. (1994), algumas características clínicas da QA podem ser os primeiros traços de malignidade associados com o carcinoma espinocelular. Porém, Lopes et al. (2015), referem que tanto as características clinicas quanto as características demográficas da QA só pode ser sugestivo de malignidade, por meio de uma biopsia para um adequado diagnóstico e tratamento.

A QA afeta principalmente homens leucodermas, tabagistas, com idade média de 61,8 anos, variando da quarta a oitava década de vida (KAUGARS et al.,1999). Estes, na história clínica, relatam exposição solar crônica e em excesso ou razões de maior vulnerabilidade à exposição, como trabalho sob o sol, morar em locais de baixa altitude, ser leucoderma e história de queimadura (MAIN e PAVONE, 1994; MARKOPOULOS et al., 2004). Eles manifestam também riscos maiores de evoluir para carcinoma de lábio (PICASCIA e ROBINSON, 1987).

Além da exposição solar, tabagismo e/ou alcoolismo, existem outras etiologias que podem colaborar para 0 aparecimento de lesões bucais, como a deficiência no sistema imune e carências nutricionais (POFFO et al, 2008). No presente relato de caso, por exemplo, a deficiência de ferro contribuiu para manifestação de queilite na cavidade bucal, o sinal clínico presente no paciente era a palidez da mucosa, e por fim, a anemia ferropriva foi confirmada através de exame de sangue. 
A anemia é uma condição comum no idoso, geralmente multifatorial e sua prevalência aumenta com a idade. Embora o declínio da hemoglobina tenha sido, no passado, considerado uma consequência normal do envelhecimento, as evidências acumuladas na literaruta demonstram que a presença de anemia nas pessoas idosas reflete saúde comprometida e aumento da vulnerabilidade para desfechos adversos (GURALNIK et al., 2004).

As causas de anemia têm sido divididas em três grandes grupos: um terço com deficiência nutricional, um terço com anemia das doenças crônicas, atualmente chamada de anemia da inflamação crônica e/ou insuficiência renal crônica, e o terço restante com anemia inexplicada (PATEL, 2008).A deficiência de ferro sozinha é responsável por quase metade dos casos de anemias causadas por deficiências nutricionais (EISENSTAEDT et al., 2006).

Diante do diagnóstico de quelite actínica associada a anemia ferropriva no presente relato de caso, foi tomado como conduta principal a reposição de ferro para a resolução da anemia e consequentemente da queilite actínica, deste modo, o paciente retornou com resolução da sintomatologia, não havendo a necessidade de biópsia.

Foi realizado este relato visando colaborar para uma melhor percepção dos profissionais de saúde a respeito das possíveis etiologias da queilite actínica, contribuindo desta forma para um correto diagnóstico, tratamento e prevenção desta lesão bucal.

\section{CONSIDERAÇÕES FINAIS}

De maneira geral, as características clínicas do caso, que foram semelhantes as encontradas na literatura, e uma boa anamnese foram fundamentais para o correto diagnóstico da lesão, impedindo sua transformação maligna. Além disso, foi possível ressaltar a importância da busca dos fatores etiológicos seja local ou sistêmico para o completo tratamento da lesão, já que a anemia ferropriva estava diretamente ligada a patologia.

\section{REFERÊNCIAS}

1. BARBOSA DL; ARRUDA IKG; DINIZ, AS. Prevalência e caracterização da anemia em idosos do Programa de Saúde da Família. Hemoter, 2006;28(4):288-292.

2. BERTINI F. Análise clínico-histológica da queilite actínica: uma abordagem interexaminadores e correlação de áreas atróficas e não atróficas, SP. Tese (Mestrado) -Faculdade de Odontologia da Universidade Estadual Paulista, São José dos Campos, 2004.

3. CAVALCANTE AS, ANBINDER AL, CARVALHO YR. Actinic cheilitis: clinical and histological features. J Oral Maxillofac Surg, 2008; 66(3):498-503.

4. CINTRA JS, TORRES SCM, SILVA MBF et al. Queilite actínica: estudo epidemiológico entre trabalhadores rurais do município de Piracaia - SP. Rev Assoc Paul Cir Dent, 2013; 67(2):118-121.

5. EISENSTAEDT R, PENNINX BW, WOODMAN RC. Anemia in the elderly: current understanding and emerging concepts. Blood Rev, 2006; 20(4):213-226.

6. GURALNIK JM, EISENSTAEDT RS, FERRUCCI $L$ et al. Prevalence of anemia in persons 65 years and older in the United States: evidence for a high rate of unexplained anemia. Blood, 2004; 104(8):2263-2268.

7. KAUGARS GE, PILLION T, SVIRSKY JA et al. Actinic cheilitis: a review of 152 cases. Oral Surg Oral Med Oral Pathol Oral Radiol Endod, 1999; 88(2):181-186.

8. LOPES ML, JÚNIOR FLS, LIMA KC et al. Clinicopathological profile and management of 161 cases of actinic cheilitis. An Bras Dermatol, 2015; 90(4):505-512.

9. MAIN JH, PAVONE M. Actinic cheilitis and carcinoma of the lip. J Can Dent Assoc, 1994;60(2):113-116.

10. MARKOPOULOS A, FARMAKI EA, KAYAVIS I. Actinic cheilitis: clinical and pathologic characteristics in 65 cases. Oral Dis, 2004;10(4):212-216. 
11. MARTINS MD, MARQUES LO, MARTINS M et al. Queilite actínica: relato de caso clínico. Conscientiae saúde, 2007; 6: 105-110.

12. NICO MMS, RIVITTI EA, LOURENÇO SV. Actinic cheilitis: histologic study of the entire vermilion and comparison with previous biopsy. J Cutan Pathol, 2007; 34(4):309-314.

13. MIRANDA AMO, FERRARI TM, CALANDRO TLL. Queilite actínica: Aspectos Clínicos e Prevalência Encontrados em uma População Rural do Interior do Brasil. Revista Saúde e Pesquisa, 2011;4(1):67-72.

14. NEVILLE B et al. Patologia Oral e Maxilofacial. Rio de Janeiro. Ed. Elsevier, 3ª edição, 2009, 340-341 p.

15. PICASCIA DD, ROBINSON JK. Actinic cheilitis: a review of the etiology, differential diagnosis and treatment. J Am Acad Dermatol, 1987;17(2):255-264.

16. PATEL KV. Epidemiology of anemia in older adults. Semin Hematol, 2008;45(4):210-7.

17. MARQUES KP, LORENÇO SV, SILVA LFF et al. Actinic Lesions in Fishermen's Lower Lip: Clinical, Cytopathological an histopathologic analysis. Clinics, 2010; 65(4):363-367.

18. POFFO, DJ; CAMARGO, AR.; SIQUEIRA, FM. Úlceras bucais relacionadas às principais desordens sistêmicas. Dissertação (Trabalho de Conclusão de Curso) - Curso de Graduação em Odontologia. Universidade Federal de Santa Catarina, Florianópolis, 2012. 117 p.

19. REGEZI JA, SCIUBBA JJ. Patologia Bucal: Correlações clínicopatológicas. 3nd ed. Rio e Janeiro, 2000; 83-84 p.

20. ROSSOE EWT, TEBCHERANI AJ, SITTART JÁ et al. Queilite actínica: avaliação comparativa estética e funcional entre as vermelhectomias clássica e em W-plastia. Anais Brasileiros de Dermatologia, 2011; 86(1):65-73.

21. TOMMASI AF, TOMMASI MH. Diagnóstico em patologia bucal.São Paulo, 2015. 